\title{
Maternal Electronic Cigarettes (e-cigs) and Offspring Asthma
}

\author{
Jacob Kraus ${ }^{1}$, Veebha Havaldar', Lauren Chiu', and Virender Rehan² \\ ${ }^{1}$ Long Beach Polytechnic High School, Long Beach, CA, USA \\ ${ }^{2}$ The Lundquist Institute, Torrance, CA, USA
}

\section{$\underline{\text { ABSTRACT }}$}

Given that the rise of electronic cigarettes (e-cigs) has become increasingly relevant to the younger generations of today's society, this paper investigates the impact of e-cig components, specifically nicotine and various e-cig flavoring chemicals, to fetal exposure during pregnancy on offspring respiratory outcomes. Previous animal studies primarily document the fetal side effects attributed to nicotine, including impaired lung development, with a model of direct chemical exposure, but we have hypothesized that in e-cig users, such phenotypes could result from and be exacerbated by the additional amalgam of chemicals that are responsible for the flavoring of e-cigs. Therefore, we have examined the harmful effects of nicotine and flavoring chemicals used in e-cigs in defense of the hypothesis that perinatal inhaled nicotine and e-cig flavoring exposure in vivo results in an airway asthmatic phenotype in offspring, which is transmitted transgenerationally, is characterized by Th2 polarization, and is more severe with combined exposure than with either constituent alone. The findings of this review support the hypothesis of this paper in regard to the potential detrimental respiratory effects of combined constituent exposure and indicate the need for the testing of further experimental animal models to better understand the foreseeable health implications of a rising e-cig use.

\section{Introduction}

In recent years, electronic cigarettes (e-cigs) have experienced a rise in users, especially among younger populations. This can primarily be attributed to from an increasingly widespread perception that e-cigs are safer than conventional cigarettes; in fact, the use of these devices can be more harmful than traditional cigarettes due to an elevated concentration of nicotine in e-cig solutions and a battery-powered vaporizer that can be heated to higher temperatures, delivering greater levels of nicotine (Spindel \& McEvoy 2016). The array of e-cig flavor options additionally plays an important role in attracting use from young users. As a young generation of reproductive-age women have been exposed to unregulated, persuasive e-cig advertisements, they are vulnerable to the harmful effects of nicotine and other e-cig flavoring chemicals on the progression of their pregnancy and health of their child. One survey of pregnant women showed $43 \%$ were unaware e-cigs contain nicotine and believed that e-cigs pose a lower risk to the fetus than traditional cigarettes (Mark et al., 2015). Another study of pregnant women found $11.9 \%$ were current e-cig users (Orzaball \& Ramadoss, 2019). Overall, it is likely that the addictive qualities of nicotine will result in an increase of their use of e-cigs throughout pregnancy, which presents a serious public health concern for current and future generations.

Considering the current and foreseeable growth in the number of e-cig users, especially those at a childbearing age, this review intends to analyze the impact of e-cig use during pregnancy to fetal lung development. Specifically, we have hypothesized that perinatal inhaled nicotine and e-cig flavoring exposure in vivo results in an airway asthmatic phenotype in offspring, which is transmitted transgenerationally, is characterized by Th2 polarization, and is more severe with combined exposure than with either constituent alone. The following review indicates the possible detrimental effects of combined perinatal nicotine and e-cig use on fetal lung development and future respiratory 
complications, and these findings are intended to guide further experimental models that evaluate this hypothesis to improve understanding and potential clinical outcomes.

\section{Combined Perinatal Exposure to Nicotine and E-Cig Flavoring}

It is possible for nicotine to cross the placenta to the fetus following 30-45 minutes after the time of intake. Traces of nicotine could stay in the amniotic fluid as a result of being contained via fetal urine, resulting in a continuous cycling and repeated exposure to the fetus. Nicotine has the potential to dramatically alter the development of the fetus, evidenced by increased levels of cotinine in the amniotic fluid. Nicotine can induce a constriction of maternal blood vessels, resulting in a deficiency of nutrients and oxygen to the developing child. The lungs and respiratory system begin to fully develop between 26 to 36 weeks, the saccular stage of lung development. Nicotine exposure during this period can alter expression of genes involved in cell proliferation, T-helper (Th) type 1 (Th1) and type 2 (Th2) immune balance, fibrosis, and viral immune responses (NCBI).

Aside from the destructive properties of nicotine within e-cigs, the flavoring components of e-cigs are also a major cause for concern. Flavor aldehydes are the main chemicals that compose the flavoring components, and they have shown to be unstable in e-cig liquids. Published in the Nicotine \& Tobacco Research Journal, a 2018 study examined how flavor aldehydes undergo chemical reactions to form novel chemical species that are harmful to e-cig users (Erythropel et al. 2018). Using gas chromatography, the researchers determined the concentrations of flavor aldehydes and reaction products in e-liquids, and in the vapor generated from commercial e-cigs. The researchers also used ultraviolet spectroscopy to monitor the stability of the detected reactions products in aqueous media and nuclear magnetic resonance spectroscopy to monitor the effects of the reaction products on irritant receptors, which were determined by fluorescent calcium imaging in HEK-293T cells. With these methods, the results showed that flavor aldehydes including cinnamaldehyde, ethylvanillin, benzaldehyde, citral, and vanillin quickly reacted with the propylene glycol (PG), the e-liquid solvent, upon mixing, and over $40 \%$ of the aldehydes were converted to flavor aldehyde PG acetals. PG acetals were also detected in commercial e-liquids, further demonstrating the presence of PG acetals in e-cigs. The vaping experiments conducted showed that $50 \%-80 \%$ of the PG acetals remained in the e-cig vapor from the initial liquid. When left in a physiological aqueous solution, the acetals remained stable with half-lives above 36 hours, suggesting that the acetals persist when inhaled by users. Examining HEK-293T cells, these acetals activated aldehyde-sensitive TRPA1 irritant receptors, and aldehyde-insensitive TRPV1 irritant receptors. With these results, it can be concluded that new compounds that have unexpected toxicological effects are formed after mixing the constituents of e-liquids, demonstrating the chemical instability of e-cig liquids, and suggesting that the combined exposure to nicotine and the flavoring components in e-cigs has worse effects than either constituent alone.

While the e-cig flavoring chemicals are detrimental when combined with nicotine and other components, the effects of the flavoring components alone have proven to be harmful as well. In a study published by the Journal of the American College of Cardiology in 2019, researchers generated endothelial cells from induced pluripotent stem (iPS) cells and exposed them to e-liquids, or serum from individuals who used e-cigs (Lee et al. 2019). The effects of six popular flavors were investigated-tobacco, sweet tobacco with caramel and vanilla, cinnamon, fruit, sweet butterscotch, and menthol-with varying nicotine levels of 0,6 , and 18 milligrams per milliliter. The researchers found that the endothelial cells were less viable and exhibited increased levels of reactive oxygen species (ROS), which are known to cause DNA damage, increased activity of caspase 3/7, which are key mediators of apoptosis, and increased uptake of low-density lipoproteins, which are commonly associated with endothelial dysfunction and inflammation. These findings, solidified by the impaired vascular tube formation and migration, confirm that endothelial dysfunction can be caused by the e-cig flavoring liquids. Additionally, the cinnamon and menthol flavors, with and without nicotine, had especially strong cytotoxic effects on the endothelial cells as compared to the other four flavors, demonstrating the variable potency of the different chemicals among flavors. Finally, regarding the impact of e-cig flavoring on pregnancy and early development through aerosols, it should be noted that aside from impaired lung growth, altered 
alveolar size, and altered inflammatory environment, as found in this study, a specific effect of the flavoring is altered global DNA methylation (Orzabal \& Ramadoss, 2019).

\section{Onset of Th2 Polarization for Asthmatic Phenotype}

Helper T type 2 (Th2) cells are believed to generate and moderate the asthamtic airway inflammatory response (Salvi et al. 2001). Th2 polarization, which refers to the proliferation of Th2 responses inversely with Th1, has been shown to drive an inherited asthmatic phenotype. Studies derived from cellular and murine models indicate the significant presence of Th2 cell polarization and an allergen-dependent response, and human studies attribute alterations in lung cytokine production coinciding with Th2 polarization to develop an inflammatory response triggering the asthmatic phenotype (Castro et al., 1999).

While it is apparent that Th2 polarization can contribute to a lung proinflammatory response, it is important to note the capability for the resulting phenotype to be transmitted across generations. Epigenetic changes constitute the mechanisms that control $\mathrm{T}$ cell activation and skewing as well as phenotype expression as a Th1 or Th2 cell (Zeng et al., 2013). When Th2 skewing occurs, which is controlled by the demethylation and histone modification of the interleukin 4 cytokine, Th2 regulators and cytokines are expressed, which subsequently silence Th1 differentiation and production (Bégin \& Nadeau, 2014). As previously mentioned, this results in a Th-2 dominant Th1/Th2 balance and cytokine milieu, contributing to the appearance of an asthamtic phenotype. Experimental testing in mice have shown that perinatal nicotine exposure stimulated Th2 cytokine production, which initiated Th2 polarization (Qiu et al. 2017). Following the development of the offspring found the presence of increased pulmonary inflammation, leading to the appearance of the asthmatic phenotype with long-term implications, including Chronic Obstructive Pulmonary Disease (COPD) and autoimmune diseases. This study suggests that nicotine acts as an immunosuppressant, which perinatally can expose a fetus to infection and illness, including respiratory complications, as observed. Therefore, it can be reasonably inferred that the epigenetic mechanisms controlling $\mathrm{Th} 2$ polarization are activated with perinatal nicotine exposure. However, the effects of the flavoring chemicals within the e-cig on Th2 polarization and the related epigenetic influence are yet to be tested and should be delineated for further research.

\section{Airway Asthmatic Phenotype}

Considering the epigenetic mechanisms initiated from the combined perinatal exposure to nicotine and e-cig flavoring chemicals and the onset of Th2 polarization, it is important to delineate the anatomical characteristics of the resulting airway asthmatic phenotype in offspring. Experimental animal models in sheep, rats, and mice display a phenotype including alterations in pulmonary structure such as an increased airway muscle deposition, increased thickness of alveolar walls, and enhanced hyper-responsive airways (Kuniyoshi \& Rehan, 2019). Additionally, an explant model study indicates the presence of lung branching morphogenesis and dysanaptic lung growth, which resulted in curved, disproportionate airways in offspring (Wongtrakool et al., 2007). Increases in collagen and alveolar volume along with decreased lung size and volume were observed in a monkey model (Orzabal \& Ramadoss, 2019). Overall, these studies demonstrate the several anatomical lung alterations that engender the airway asthmatic phenotype, which evidently places offspring at a greater risk for future respiratory complications.

\section{Evidence of Transgenerational Epigenetic Phenotype Inheritance}

Multiple studies display animal models indicative that environmentally induced phenotypes are transgenerationally transmitted. In one study, plastic derived endocrine disruptors (BPA) were tested on female rats to evaluate epigenetic transgenerational inheritance of obesity, reproductive disease, and sperm epimutations (Manikkam, 2013). F0 generation female rats received daily injections of mixed plastic compounds during days 8 to 14 of fetal gestation, and the 
appearance of adult disease was analyzed in the F1 and F3 generation rats. In an evaluation of the F3 generation, obesity and reproductive abnormalities, including pubertal differences, testis disease, and ovarian disease from ovarian insufficiency and polycystic ovaries, were observed. Furthermore, analysis of the F3 generation sperm epigenome showed 197 regions of DNA methylation that contributed to the transmission of epimutations across generations that led to the inheritance of these offspring traits. In addition, a more recent study utilized a mouse model of Fetal Alcohol Spectrum Disorders (FASD) to identify the transgenerational effects of prenatal ethanol exposure on neocortical development (Abbott et al. 2017). Experimental dams of the F0 generation were administered an ethanol solution from the beginning of copulatory plug detection (gestational day 0.5) to the day of birth (gestational day 19.5). Observation of the F1 to the F3 generation resulted in transgenerational alterations including decreases noticed in body weight, brain weight, and cortical length, and changes in cortical connectivity. Behavioral testing across each generation displayed a consistent presence of anxious and depressive behaviors as well as reduced motor function that was transgenerationally-inherited. Statistical analysis of epigenetic markers indicated the significant presence of DNA methylation and global hydroxymethylation in F1 to F3 offspring. Both studies are evident of the environmentally-induced epigenetics that contribute to the transgenerational transmission of phenotypes.

Evaluating transgenerational epigenetic inheritance of phenotypes in humans has received limited research attention due to the clinical complexities to conduct such a study, but one historical study spurred on by an environmental tragedy offers a glimpse to how epigenetic inheritance across generations in humans comes to fruition. The Dutch Winter Hunger Study investigates the impact of a mid-20th century famine in The Netherlands on offspring health of pregnant women (Schulz, 2010). The stage of gestation during parental exposure to famine determined the extent of the future health impact to the fetus. For instance, those exposed during late gestation were born small and maintained this stature throughout their life compared to those exposed during the early and middle stages of gestation, who displayed several abnormal phenotypes including heart disease, altered lipid profiles, obesity, and obstructive airways. As this study followed offspring into their adulthood, researchers later found that food deficiency programmed alterations in placental growth through introducing DNA methylation in adult female offspring, who had been exposed to famine in utero. In conjunction with this review's hypothesis, this study supports the concept that epigenetic mechanisms in a human model can contribute to the transmission of inheritable phenotypes across generations. While this study solely offered a multigenerational perspective on the inherited phenotypes to offspring, it is important to note that this study is currently ongoing to evaluate the resulting health of the future generations of the ancestral generation initially exposed to this famine, which means the transgenerationally transmitted phenotypes in further offspring generations are being recorded at the time of this paper. Nonetheless, animal experiments suggest nutritional changes occurring exclusively during oocyte development and early gestation can permanently alter gene methylation status and their succeeding expression, indicating epimutations in the F1 generation are potent to be transmitted transgenerationally and proliferate abnormal phenotypes (Oliver et al., 2005).

A limited number of animal studies have evaluated the transgenerational transmission of the airway asthmatic phenotype from perinatal nicotine exposure. Epigenetic inheritance of the asthmatic phenotype in a rat model was observed in the F1 and F2 generations after gestational nicotine exposure in the F0 generation (Liu et al., 2020). DNA methylation changes environmentally induced from nicotine exposure increased with the asthmatic phenotype progressively more severe in the F2 offspring compared to the preceding generations. Although the impact of e-cig flavoring chemicals on fetal development has been documented, a model evaluating the severity of combined perinatal exposure to nicotine and e-cig flavoring chemicals has not been tested. Therefore, as more individuals become inclined to transition to using e-cigs, this model is potent towards providing an experimental outlook that can gauge the clinical outcome from combined fetal exposure to these chemical constituents.

\section{Discussion of Hypothesis}

This review demonstrates the potency of perinatal nicotine and e-cig flavoring exposure on inducing negative transgenerational respiratory outcomes in offspring. The described animal studies support the unfavorable results 
nicotine and e-cig chemicals could pose as constituents in provoking epigenome alterations that lead to the consequential respiratory phenotypes in succeeding generations. Epigenetic mechanisms, including DNA methylation and histone modifications, are activated during gestational exposure to these constituents, altering normal fetal development in this stage of pregnancy. Specifically, these mechanisms initiate T cell proliferation of the Th2 phenotype and the interleukin 4 cytokine. As a result, Th2 polarization from a Th1/Th2 imbalance and a significant cytokine environment gain presence, where both are key factors in engendering an inflammatory airway response during fetal lung development that drives the resulting asthmatic phenotype. Increase airway and alveolar thickness, abnormal lung branching, and decreased lung size characterize this offspring phenotype, which combined constituent exposure can further exacerbate. As the epigenetic mechanisms that germinate this phenotype can evidently enable it to be transmitted transgenerationally, e-cig use in those at child-bearing age supports the risk for their continued use during future pregnancies, leading to potential complications and adverse offspring outcomes that can persist for several generations.

\section{Proposed Model for Further Research}

Past examinations of models intended to replicate smoking exposure to test subjects helped us determine how to simulate e-cig exposure to the fetus. The first choice was a monkey or baboon model, given the similarity of respiratory development in utero, but this was eliminated due to the staggering cost and foreseeable issues with ventilation and maintenance. Another model that was considered was the C. elegans model, which was discouraged due to its inability to present an appropriate examination of the airway's phenotype. A possibility that was entertained for a period of time was a rabbit model, but this was eliminated as well after the consideration of the necessary similarity in lung development to humans.

After considering that this characteristic is found in rodents, we decided to recommend a nonhuman rat model, feeling it was most appropriate and efficient for our purposes. This conclusion was reached after taking into account both the financial advantages and the average 21- to 23-day gestation period of the Sprague-Dawley rat, one of the most used in biomedical research (Brower et al. 2015).

We conducted searches within scholarly articles and published studies, eventually finding a previously established rat model for cigarette smoke exposure (CSE) (Ypsilantis et al. 2012). This system, meant to expose rats to pure cigarette smoke contained a ventilator (Veterinary anesthesia ventilator model 2000, Hallowell EMC, Pittsfield, MA), an acrylic smoke-generating chamber (5430 cubic centimeters), and an acrylic white whole-body CSE chamber (200,000 cubic centimeters), serially adjoined with silicone tubes interlined by Heimlich valves (Becton Dickinson, Franklin Lakes, NJ). The ventilator was set to provide a set quantity of clean air after a set number of seconds. The smoke-generating chamber contained one constantly lit tar cigarette, which produced the smoke that was then delivered to the CSE chamber, where the exposed rats were placed, and exhausted through a hole.

While this is in itself a highly effective model, for the purposes of this study we would replace the cigarette smoke with the constituents being tested in our tested hypothesis: nicotine vapor and e-cig flavoring vapor. Another discrepancy our study has from the established model is the planned exposure of pregnant rats to the toxins, which will be done for a shorter period of time and quantities due to the weights and the gestation period of the SpragueDawley rats. We would use the CSE model to expose the pregnant rats of the F0 generation to side stream e-cig nicotine vapor, flavoring vapor, and toxin free vapor as a control, as opposed to the tar cigarette smoke it was originally designed for.

The average human female gestation period is 36 weeks, and that of the female Sprague-Dawley rat is 3 weeks. We used this information to determine the amount of time we should expose the pregnant rats to the CSE system, factoring the time periods down by 12. First assuming a human mother using e-cigarettes would consume the average amount of liquid per day, 3-5 mL, we can extrapolate that daily use will add up to 756-1260 mL (averaged to $1.008 \mathrm{~L}$ or $1008 \mathrm{~mL}$ ) during the pregnancy. We divided this by twelve, to get that the Sprague-Dawley rat would consume $84 \mathrm{~mL}$ of fluid over its pregnancy. We then took into account the weights of the rats in relation to those of 
women. The average woman weighs 166 pounds, compared to the average rat weight 0.5 pounds, giving us a factor of 332. We finally arrived at the conclusion that the pregnant rat would consume a total of $253 \mu \mathrm{L}$ during pregnancy, approximately $11.5 \mu \mathrm{L}$ per day. In many liquids, total flavor chemicals were found to be in the $\sim 1-4 \%$ range (10-40 $\mathrm{mg} / \mathrm{mL}$ ); labelled levels of nicotine were in the range of $0.6-2.4 \%$ (6 to $24 \mathrm{mg} / \mathrm{mL}$ ), according to Tobacco Control. We can translate this to the study by concluding that the rats in the nicotine group would be administered approximately $0.1725 \mu \mathrm{L}$ nicotine in vapor per day, while the rats in the flavoring group would be administered approximately $0.2875 \mu \mathrm{L}$ flavoring in vape per day. Using the CSE model, the pregnant rats in either group would be exposed to these quantities of toxins via dilution in vapor.

Once the pregnant rats are to give birth, the pups (F1 generation) would be fed Ad libitum until they were to reproduce as well. This would be done with the traditional protocol for breeding laboratory rats. When the F2 generation is produced, their pulmonary systems are to be examined via a physical dissection to determine the extent of the impairment. This could also be done via a sedation, in which the pups were to be anesthetized and sedated with ketamine $(70 \mathrm{mg} / \mathrm{kg})$ and xylazine $(7 \mathrm{mg} / \mathrm{kg})$. Additionally, the examination of the airway would be supplemented with a check for Th2 polarization, which was established as a distinguishing factor for the airway asthmatic phenotype. Given that this typically occurs in the secondary lymphoid organs (Kaiko et al., 2008), they will be examined for this during the proposed inspection.

\section{Conclusion}

As presented in this paper, there is preliminary evidence to believe that perinatal inhaled nicotine and e-cig flavoring exposure in vivo results in an airway asthmatic phenotype in offspring, which is transmitted transgenerationally, is characterized by Th2 polarization, and is more severe with combined exposure than with either constituent alone. Since it has been shown that many mothers use e-cigs during pregnancy, either out of negligence or misunderstanding, this is a great cause for concern for the potential impacts of the nicotine and flavoring components on the developing fetus. With this in mind, we highly recommend the proposed animal model presented above to study the effects of perinatal inhaled e-cig component exposure. While there is supporting evidence to believe in the validity of the hypothesis, further experimental research is needed to draw accurate conclusions.

\section{Acknowledgements}

We would like to thank Dr. Virender Rehan and The Lundquist Institute for Biomedical Innovation for guidance on the composition of this review.

\section{References}

Abbott CW, Rohac DJ, Bottom RT, Patadia S, Huffman KJ. Prenatal Ethanol Exposure and Neocortical Development: A Transgenerational Model of FASD. Cerebral Cortex. 2017;28(8):2908-2921. https://doi.org/10.1093/cercor/bhx 168 .

Bégin P, Nadeau KC. Epigenetic regulation of asthma and allergic disease. Allergy Asthma Clin Immunol. 2014;10(1):27.

Brower M, Grace M, Kotz CM, Koya V. Comparative analysis of growth characteristics of Sprague Dawley rats obtained from different sources. Laboratory Animal Research. 2015;31(4):166. 
Castro M, Chaplin DD, Walter MJ, Holtzman MJ. Could Asthma Be Worsened by Stimulating the T-helper Type 1 Immune Response? American Journal of Respiratory Cell and Molecular Biology. 2000;22(2):143-146. https://doi.org/10.1165/ajrcmb.22.2.f174.

Erythropel H, Jabba SV, DeWinter TM, Mendizabal M, Anastas PT, Jordt SE, Zimmerman JB. Formation of Flavorant-Propylene Glycol Adducts With Novel Toxicological Properties in Chemically Unstable ECigarette Liquids. Nicotine \& Tobacco Research. 2018;21(9):1248-1258. https://doi.org/10.1093/ntr/nty192.

Kaiko, Gerard E, et al. Immunological Decision-Making: How Does the Immune System Decide to Mount a Helper T-Cell Response? Immunology. Mar. 2008. https://doi.org/10.1111/j.1365-2567.2007.02719.x.

Kuniyoshi KM, Rehan VK. The impact of perinatal nicotine exposure on fetal lung development and subsequent respiratory morbidity. Birth Defects Research. 2019;1-14. https://doi.org/10.1002/bdr2.1595.

Lee WH, Ong S, Zhou Y, Tian L, Bae HR, Baker N, Whitlatch A, Mohammadi L, Guo H, Nadeau KC, et al. Modeling Cardiovascular Risks of E-Cigarettes With Human-Induced Pluripotent Stem Cell-Derived Endothelial Cells. Journal of the American College of Cardiology. 2019;73(21):2722-2737. https://doi.org/10.1016/j.jacc.2019.03.476.

Liu J, Yu C, Doherty TM, Akbari O, Allard P, Rehan VK. Perinatal nicotine exposure-induced transgenerational asthma: Effects of reexposure in F1 gestation. The FASEB Journal. 2020;00:1-16. https://doi.org/10.1096/fj.201902386R.

Manikkam M, Tracey R, Guerrero-Bosagna C, Skinner MK. Plastics derived endocrine disruptors (BPA, DEHP and DBP) induce epigenetic transgenerational inheritance of obesity, reproductive disease and sperm epimutations. PLoS One. 2013;8(1):e55387. https://doi.org/10.1371/journal.pone.0055387.

Mark KS, Farquhar B, Chisolm MS, Coleman-Cowger VH, Terplan M. Knowledge, attitudes, and practice of electronic cigarette use among pregnant women. J Addict Med 2015;9:266-272. https://doi.org/10.1097/ADM.0000000000000128.

Oliver MH, Hawkins P, Harding JE. Periconceptional Undernutrition Alters Growth Trajectory and Metabolic and Endocrine Responses to Fasting in Late-Gestation Fetal Sheep. Pediatric Research. 2005;57(4):591-598. https://doi.org/10.1203/01.PDR.0000155942.18096.9C.

Orzabal M, Ramadoss J. Impact of Electronic Cigarette Aerosols on Pregnancy and Early Development. Current Opinion in Toxicology. 2019;14:14-20. https://doi.org/10.1016/j.cotox.2019.05.001.

Qiu F, Liang C, Liu H, Zeng Y, Hou S, Huang S, Lai X, Dai Z. Impacts of cigarette smoking on immune responsiveness: Up and down or upside down?. Oncotarget. 2017;8(1):268-284. https://doi.org/10.18632/oncotarget.13613.

Salvi SS, Suresh Babu K, Holgate ST. Is Asthma Really Due to a Polarized T Cell Response Toward a Helper T Cell Type 2 Phenotype? American Journal of Respiratory and Critical Care Medicine. 2001;164(8):1343-1346. https://doi.org/10.1164/ajrccm.164.8.2103080.

Schulz LC. The Dutch Hunger Winter and the Developmental Origins of Health and Disease. Proceedings of the National Academy of Sciences. 2010;107(39):16757-16758. https://doi.org/10.1073/pnas.1012911107. 
Spindel ER, McEvoy CT. The Role of Nicotine in the Effects of Maternal Smoking during Pregnancy on Lung Development and Childhood Respiratory Disease. Implications for Dangers of E-Cigarettes. American Journal of Respiratory and Critical Care Medicine. 2019;193:486-494. https://doi.org/10.1164/rccm.201510-2013PP.

Wongtrakool C, Roser-Page S, Rivera HN, Roman J. Nicotine alters lung branching morphogenesis through the alpha7 nicotinic acetylcholine receptor. Am J Physiol Lung Cell Mol Physiol. 2007;293(3):L611-L618. https://doi.org/10.1152/ajplung.00038.2007.

Ypsilantis P, Politou M, Anagnostopoulos C, Kortsaris A, Simopoulos C. A Rat Model of Cigarette Smoke Abuse Liability. Comparative Medicine, American Association for Laboratory Animal Science. 2012;62(5):395-399.

Zeng WP. 'All things considered': transcriptional regulation of T helper type 2 cell differentiation from precursor to effector activation. Immunology. 2013;140(1):31-38. 